\title{
COMPARATIVE GROWTH ANALYSIS OF SPECIAL TYPE OF DIFFERENTIAL POLYNOMIAL GENERATED BY ENTIRE AND MEROMORPHIC FUNCTIONS ON THE BASIS OF THEIR $(p, q)$-th ORDER
}

\author{
Tanmay Biswas \\ Rajbari, Rabindrapalli, R. N. Tagore Road \\ P.O.- Krishnagar, Dist.-Nadia, PIN - 741101 \\ West Bengal, INDIA
}

\begin{abstract}
In this paper we aim to establish some results depending on the comparative growth properties of composite transcendental entire or meromorphic functions and some special type of differential polynomials generated by one of the factors on the basis of $(p, q)$-th order and $(p, q)$-th lower order where $p, q$ are positive integers with $p \geq q$.
\end{abstract}

AMS Subject Classification: 30D30, 30D35

Key Words: entire function, meromorphic function, $(p, q)$-th order, $(p, q)$-th lower order, composition, growth, special type of differential polynomial

\section{Introduction, Definitions and Notations}

Let us consider that the reader is familiar with the fundamental results and the standard notations of the Nevanlinna theory of meromorphic functions which are available in $[5,9,11,12]$. We also use the standard notations and definitions of the theory of entire functions which are available in [13] and therefore we do not explain those in details. For $x \in[0, \infty)$ and $k \in \mathbb{N}$, we define $\exp ^{[k]} x=\exp \left(\exp ^{[k-1]} x\right)$ and $\log ^{[k]} x=\log \left(\log ^{[k-1]} x\right)$ where $\mathbb{N}$ be the set of all positive integers. Let $f$ be an entire function defined in the open complex 
plane $\mathbb{C}$. The maximum modulus function $M_{f}(r)$ corresponding to $f$ is defined on $|z|=r$ as $M_{f}(r)=\max |z|=r|f(z)|$. When $f$ is meromorphic, one may introduce another function $T_{f}(r)$ known as Nevanlinna's characteristic function of $f$, playing the same role as $M_{f}(r)$. However, the Nevanlinna Characteristic function of a meromorphic function $f$ is defined as

$$
T_{f}(r)=N_{f}(r)+m_{f}(r),
$$

wherever the function $N_{f}(r, a)\left(\bar{N}_{f}(r, a)\right)$ known as counting function of $a$ points (distinct $a$-points) of meromorphic $f$ is defined as follows:

$$
\begin{gathered}
N_{f}(r, a)=\int_{0}^{r} \frac{n_{f}(t, a)-n_{f}(0, a)}{t} d t+\bar{n}_{f}(0, a) \log r \\
\left(\bar{N}_{f}(r, a)=\int_{0}^{r} \frac{\overline{n_{f}}(t, a)-\bar{n}_{f}(0, a)}{t} d t+\bar{n}_{f}(0, a) \log r\right),
\end{gathered}
$$

in addition we represent by $n_{f}(r, a)\left(\overline{n_{f}}(r, a)\right)$ the number of $a$-points (distinct $a$-points) of $f$ in $|z| \leq r$ and an $\infty$-point is a pole of $f$. In many occasions $N_{f}(r, \infty)$ and $\bar{N}_{f}(r, \infty)$ are symbolized by $N_{f}(r)$ and $\bar{N}_{f}(r)$, respectively.

On the other hand, the function $m_{f}(r, \infty)$ alternatively indicated by $m_{f}(r)$ known as the proximity function of $f$ is defined as:

$$
\begin{aligned}
& m_{f}(r)=\frac{1}{2 \pi} \int_{0}^{2 \pi} \log ^{+}\left|f\left(r e^{i \theta}\right)\right| d \theta, \quad \text { where } \\
& \log ^{+} x=\max (\log x, 0) \text { for all } x \geqslant 0 .
\end{aligned}
$$

Also we may employ $m\left(r, \frac{1}{f-a}\right)$ by $m_{f}(r, a)$.

If $f$ is entire, then the Nevanlinna Characteristic function $T_{f}(r)$ of $f$ is defined as

$$
T_{f}(r)=m_{f}(r) .
$$

Further, let $n_{0}, n_{1}, n_{2}, \ldots n_{k}$ be non negative integers. For a transcendental meromorphic function $f$, we call the expression $M[f]=f^{n_{0}}\left(f^{(1)}\right)^{n_{1}}\left(f^{(2)}\right)^{n_{2}} \ldots$ $\left(f^{(k)}\right)^{n_{k}}$ to be a monomial generated by $f$. The numbers $\gamma_{M}=n_{0}+n_{1}+n_{2}+$ $\ldots+n_{k}$ and $\Gamma_{M}=n_{0}+2 n_{1}+3 n_{2}+\ldots+(k+1) n_{k}$ are called respectively the degree and weight of the monomial. If $M_{1}[f], M_{2}[f], \ldots, M_{n}[f]$ denote monomials in $f$, then

$$
Q[f]=a_{1} M_{1}[f]+a_{2} M_{2}[f]+\ldots+a_{n} M_{n}[f]
$$


where $a_{i} \neq 0(i=1,2, \ldots, n)$ is called a differential polynomial generated by $f$ of degree $\gamma_{Q}=\max \left\{\gamma_{M_{j}}: 1 \leq j \leq n\right\}$ and weight $\Gamma_{Q}=\max \left\{\Gamma_{M_{J}}: 1 \leq j \leq n\right\}$. Also we call the numbers $\underline{\gamma_{Q}}=\min _{1 \leq j \leq s} \gamma_{M j}$ and $k$ (the order of the highest derivative of $f$ ) the lower degree and the order of $Q[f]$, respectively. If $\underline{\gamma_{Q}}=\gamma_{Q}$, $Q[f]$ is called a homogeneous differential polynomial.

However, the ratio $\frac{T_{f}(r)}{T_{g}(r)}$ as $r \rightarrow \infty$ is called the growth of $f$ with respect to $g$ in terms of the Nevanlinna Characteristic functions of the meromorphic functions $f$ and $g$. Moreover, the order $\rho_{f}$ (resp. lower order $\lambda_{f}$ ) of an entire function $f$ which is generally used in computational purpose is defined as

$$
\rho_{f}=\varlimsup_{r \rightarrow \infty} \frac{\log \log M_{f}(r)}{\log r}\left(\operatorname{resp} . \lambda_{f}=\varliminf_{r \rightarrow \infty} \frac{\log \log M_{f}(r)}{\log r}\right) .
$$

If $f$ is a meromorphic function, then

$$
\rho_{f}=\varlimsup_{r \rightarrow \infty} \frac{\log T_{f}(r)}{\log r}\left(\text { resp. } \lambda_{f}=\varliminf_{r \rightarrow \infty} \frac{\log T_{f}(r)}{\log r}\right) .
$$

Extending this notion, Juneja et. al. [6] defined the $(p, q)$-th order (resp. $(p, q)$-th lower order) of an entire function $f$ for any two positive integers $p, q$ with $p \geq q$ which is as follows:

$$
\rho_{f}(p, q)=\varlimsup_{r \rightarrow \infty} \frac{\log ^{[p]} M_{f}(r)}{\log ^{[q]} r}\left(\operatorname{resp} . \lambda_{f}(p, q)=\varliminf_{r \rightarrow \infty} \frac{\log ^{[p]} M_{f}(r)}{\log ^{[q]} r}\right) .
$$

If $f$ is meromorphic function, then

$$
\rho_{f}(p, q)=\varlimsup_{r \rightarrow \infty} \frac{\log ^{[p-1]} T_{f}(r)}{\log ^{[q]} r} \text { and } \lambda_{f}(p, q)=\varliminf_{r \rightarrow \infty} \frac{\log ^{[p-1]} T_{f}(r)}{\log ^{[q]} r},
$$

where $p, q$ are any two positive integers with $p \geq q$.

These definitions extend the generalized order $\rho_{f}^{[l]}$ and generalized lower order $\lambda_{f}^{[l]}$ of an entire function $f$ considered in [10] for each integer $l \geq 2$ since these correspond to the particular case $\rho_{f}^{[l]}=\rho_{f}(l, 1)$ and $\lambda_{f}^{[l]}=\lambda_{f}(l, 1)$. Clearly, $\rho_{f}(2,1)=\rho_{f}$ and $\lambda_{f}(2,1)=\lambda_{f}$.

An entire or meromorphic function for which $(p, q)$-th order and $(p, q)$-th lower order are the same is said to be of regular $(p, q)$-growth. Functions which are not of regular $(p, q)$-growth are said to be of irregular $(p, q)$-growth.

In this connection we just recall the following two definitions which will be needed in the sequel. 
Definition 1. A function $\rho_{f}^{[l]}(r)$ is called a generalized proximate order of a meromorphic function $f$ relative to $T_{f}(r)$ if:

(i) $\rho_{f}^{[l]}(r)$ is non-negative and continuous for $r \geqslant r_{0}$, say,

(ii) $\rho_{f}^{[l]}(r)$ is differentiable for $r \geqslant r_{0}$ except possibly at isolated points at which $\rho_{f}^{[l] \prime}(r+0)$ and $\rho_{f}^{[l] \prime}(r-0)$ exist,

(iii) $\lim _{r \rightarrow \infty} \rho_{f}^{[l]}(r)=\rho_{f}^{[l]}<\infty$,

(iv) $\lim _{r \rightarrow \infty} \rho_{f}^{[l] \prime}(r) \prod_{i=0}^{l-1} \log [i] r=0$ and

(v) $\varlimsup_{r \rightarrow \infty} \frac{\log ^{[l-2]} T_{f}(r)}{r^{\rho_{f}^{[l]}(r)}}=1$.

The existence of such a proximate order is proved by Lahiri [8].

Similarly one can define the generalized lower proximate order of a meromorphic function $f$ in the following way:

Definition 2. A function $\lambda_{f}^{[l]}(r)$ is defined as a generalized lower proximate order of a meromorphic function $f$ relative to $T_{f}(r)$ if:

(i) $\lambda_{f}^{[l]}(r)$ is non-negative and continuous for $r \geqslant r_{0}$, say,

(ii) $\lambda_{f}^{[l]}(r)$ is differentiable for $r \geqslant r_{0}$ except possibly at isolated points at which $\lambda_{f}^{[l] \prime}(r+0)$ and $\lambda_{f}^{[l] \prime}(r-0)$ exist,

(iii) $\lim _{r \rightarrow \infty} \lambda_{f}^{[l]}(r)=\lambda_{f}^{[l]}<\infty$,

(iv) $\lim _{r \rightarrow \infty} \lambda_{f}^{[l] \prime}(r) \prod_{i=0}^{l-1} \log ^{[i]} r=0$ and

(v) $\varliminf_{r \rightarrow \infty} \frac{\log ^{[l-2]} T_{f}(r)}{r^{\lambda_{f}^{[l]}(r)}}=1$.

In this paper we aim to establish some results depending on the comparative growth properties of composite transcendental entire or meromorphic functions and some special type of differential polynomials generated by one of the factors on the basis of $(p, q)$-th order $((p, q)$-th lower order $)$ and proximate order (proximate lower order) where $p, q$ are positive integers with $p \geq q$.

\section{Lemmas}

In this section we present some lemmas which will be needed in the sequel. 
Lemma 3. ([1]) If $f$ is a meromorphic function and $g$ is an entire function then for all sufficiently large positive numbers of $r$,

$$
T_{f \circ g}(r) \leqslant\{1+o(1)\} \frac{T_{g}(r)}{\log M_{g}(r)} T_{f}\left(M_{g}(r)\right) .
$$

Lemma 4. ([2]) Suppose that $f$ is a meromorphic function and $g$ be an entire function and suppose that $0<\mu<\rho_{g} \leq \infty$. Then for a sequence of values of $r$ tending to infinity,

$$
T_{f \circ g}(r) \geq T_{f}\left(\exp \left(r^{\mu}\right)\right)
$$

Lemma 5. ([4]) Let $g$ be an entire function. Then for any $\delta(>0)$ the function $r^{\lambda_{g}^{[l]}+\delta-\lambda_{g}^{[l]}(r)}$ is ultimately an increasing function of $r$.

Lemma 6. ([4]) Let $g$ be an entire function. Then for any $\delta(>0)$ the function $r^{\rho_{g}^{[l]}+\delta-\rho_{g}^{[l]}(r)}$ is ultimately an increasing function of $r$.

Lemma 7. ([3]) Let $f$ be a transcendental meromorphic function and $F=f^{n} Q[f]$ where $Q[f]$ is a differential polynomial in $f$, then for any $n \geq 1$,

$$
\begin{aligned}
T_{f}(r) & =O\left\{T_{F}(r)\right\} \text { as } r \rightarrow \infty \\
\text { and } T_{F}(r) & =O\left\{T_{f}(r)\right\} \text { as } r \rightarrow \infty .
\end{aligned}
$$

Lemma 8. Let $f$ be a transcendental meromorphic function and $F=$ $f^{n} Q[f]$ where $Q[f]$ is a differential polynomial in $f$, then for any $n \geq 1$

$$
\rho_{F}(p, q)=\rho_{f}(p, q) \text { and } \lambda_{F}(p, q)=\lambda_{f}(p, q) .
$$

Proof. Let us consider that $\alpha$ and $\beta$ be any two constant greater than 1 . Now we get from Lemma 7 for all sufficiently large values of $r$ that

$$
T_{F}(r)<\alpha \cdot T_{f}(r)
$$

and

$$
T_{f}(r)<\beta \cdot T_{F}(r) .
$$

Now from (1) it follows for all sufficiently large values of $r$ that

$$
\begin{aligned}
\log ^{[p]} T_{F}(r) & <\log ^{[p]} T_{f}(r)+O(1) \\
\text { i.e., } \frac{\log ^{[p]} T_{F}(r)}{\log ^{[q]} r} & <\frac{\log ^{[p]} T_{f}(r)+O(1)}{\log ^{[q]} r}
\end{aligned}
$$




$$
\text { i.e., } \rho_{F}(p, q) \leq \rho_{f}(p, q) \text {. }
$$

Again from (2) we obtain for all sufficiently large values of $r$ that

$$
\begin{aligned}
\log ^{[p]} T_{f}(r) & <\log ^{[p]} T_{F}(r)+O(1) \\
\text { i.e., } \frac{\log ^{[p]} T_{f}(r)}{\log ^{[q]} r} & <\frac{\log ^{[p]} T_{F}(r)+O(1)}{\log ^{[q]} r} \\
\text { i.e., } \quad \rho_{f}(p, q) & \leq \rho_{F}(p, q) .
\end{aligned}
$$

Therefore from (3) and (4), we get that

$$
\rho_{F}(p, q)=\rho_{f}(p, q) \text {. }
$$

In a similar manner, $\lambda_{F}(p, q)=\lambda_{f}(p, q)$.

Thus the lemma follows.

\section{Main Results}

In this section we present the main results of the paper.

Theorem 9. Let $f$ be a transcendental meromorphic function and $g$ be an entire function such that $\rho_{g}(m, n)<\lambda_{f}(p, q) \leq \rho_{f}(p, q)<\infty$, where $p, q, m, n$ are positive integers with $p \geq q, m \geq n$. Also let $F=f^{\alpha} Q[f]$, where $Q[f]$ is a differential polynomial in $f$, then for any $\alpha \geq 1$

and

$$
\text { (i) } \lim _{r \rightarrow \infty} \frac{\log ^{[p-1]} T_{f \circ g}\left(\exp ^{[n-1]} r\right)}{\log ^{[p-2]} T_{F}\left(\exp ^{[q-1]} r\right)}=0 \quad \text { if } \quad q \geq m
$$

$$
\text { (ii) } \lim _{r \rightarrow \infty} \frac{\log ^{[p+m-q-2]} T_{f \circ g}\left(\exp ^{[n-1]} r\right)}{\log ^{[p-2]} T_{F}\left(\exp ^{[q-1]} r\right)}=0 \quad \text { if } \quad q<m \text {. }
$$

Proof. Since $\rho_{g}(m, n)<\lambda_{f}(p, q)$ we can choose $\varepsilon(>0)$ is such a way that

$$
\rho_{g}(m, n)+\varepsilon<\lambda_{f}(p, q)-\varepsilon .
$$

As $T_{g}(r) \leq \log ^{+} M_{g}(r)\{\mathrm{cf}$. [5]\}, we have from Lemma 3, for all sufficiently large values of $r$ that

$$
\begin{aligned}
& \log ^{[p-1]} T_{f \circ g}\left(\exp ^{[n-1]} r\right) \leq \log { }^{[p-1]} T_{f}\left(M_{g}\left(\exp ^{[n-1]} r\right)\right)+O(1) \\
& \text { i.e., } \quad \log ^{[p-1]} T_{f \circ g}\left(\exp ^{[n-1]} r\right)
\end{aligned}
$$




$$
\leqslant\left(\rho_{f}(p, q)+\varepsilon\right) \log ^{[q]} M_{g}\left(\exp ^{[n-1]} r\right)+O(1)
$$

Now the following two cases may arise.

Case I. Let $q \geqslant m$. Then we have from (6) for all sufficiently large values of $r$ that

$$
\begin{aligned}
\log ^{[p-1]} T_{f \circ g} & \left(\exp ^{[n-1]} r\right) \\
& \leqslant\left(\rho_{f}(p, q)+\varepsilon\right) \log ^{[m-1]} M_{g}\left(\exp ^{[n-1]} r\right)+O(1) .
\end{aligned}
$$

Again for all sufficiently large values of $r$,

$$
\begin{gathered}
\log ^{[m]} M_{g}\left(\exp ^{[n-1]} r\right) \leqslant\left(\rho_{g}(m, n)+\varepsilon\right) \log ^{[n]} \exp ^{[n-1]} r \\
\text { i.e. }, \log ^{[m-1]} M_{g}\left(\exp ^{[n-1]} r\right) \leqslant r^{\left(\rho_{g}(m, n)+\varepsilon\right)} .
\end{gathered}
$$

Now from (7) and (8) we have for all sufficiently large values of $r$ that

$$
\log ^{[p-1]} T_{f \circ g}\left(\exp ^{[n-1]} r\right) \leqslant\left(\rho_{f}(p, q)+\varepsilon\right) r^{\left(\rho_{g}(m, n)+\varepsilon\right)}+O(1) .
$$

Case II. Let $q<m$. Then for all sufficiently large values of $r$ we get from (6) that

$$
\begin{aligned}
\log ^{[p-1]} & T_{f \circ g}\left(\exp ^{[n-1]} r\right) \\
& \leqslant\left(\rho_{f}(p, q)+\varepsilon\right) \exp ^{[m-q]} \log ^{[m]} M_{g}\left(\exp ^{[n-1]} r\right)+O(1) .
\end{aligned}
$$

Again for all sufficiently large values of $r$,

$$
\begin{aligned}
\log ^{[m]} M_{g}\left(\exp ^{[n-1]} r\right) & \leqslant\left(\rho_{g}(m, n)+\varepsilon\right) \log { }^{[n]} \exp ^{[n-1]} r \\
\text { i.e., } \log ^{[m]} M_{g}\left(\exp ^{[n-1]} r\right) & \leqslant \log r^{\rho_{g}(m, n)+\varepsilon} \\
\text { i.e., } \exp ^{[m-q]} \log ^{[m]} M_{g}\left(\exp ^{[n-1]} r\right) & \leqslant \exp ^{[m-q]} \log r^{\rho_{g}(m, n)+\varepsilon} \\
\text { i.e., } \exp ^{[m-q]} \log ^{[m]} M_{g}\left(\exp ^{[n-1]} r\right) & \leqslant \exp ^{[m-q-1]} r^{\rho_{g}(m, n)+\varepsilon} .
\end{aligned}
$$

Now from (10) and (11) we have for all sufficiently large values of $r$ that

$$
\begin{gathered}
\log ^{[p-1]} T_{f \circ g}\left(\exp ^{[n-1]} r\right) \leq\left(\rho_{f}(p, q)+\varepsilon\right) \exp ^{[m-q-1]} r^{\rho_{g}(m, n)+\varepsilon}+O(1) \\
\text { i.e., } \log ^{[p]} T_{f \circ g}\left(\exp ^{[n-1]} r\right) \leqslant \exp ^{[m-q-2]} r^{\rho_{g}(m, n)+\varepsilon}+O(1)
\end{gathered}
$$




$$
\begin{aligned}
& \text { i.e., } \quad \log ^{[p+m-q-2]} T_{f \circ g}\left(\exp ^{[n-1]} r\right) \\
& \leqslant \log ^{[m-q-2]} \exp ^{[m-q-2]} r^{\rho_{g}(m, n)+\varepsilon}+O(1) \\
& \text { i.e., } \log ^{[p+m-q-2]} T_{f \circ g}\left(\exp ^{[n-1]} r\right) \leqslant r^{\rho_{g}(m, n)+\varepsilon}+O(1) \text {. }
\end{aligned}
$$

Again for all sufficiently large values of $r$, we get in view of Lemma 8 that

$$
\begin{aligned}
& \log ^{[p-1]} T_{F}\left(\exp ^{[q-1]} r\right) \geqslant\left(\lambda_{F}(p, q)-\varepsilon\right) \log ^{[q]} \exp ^{[q-1]} r \\
& \text { i.e., } \log { }^{[p-1]} T_{F}\left(\exp ^{[q-1]} r\right) \geqslant\left(\lambda_{f}(p, q)-\varepsilon\right) \log r \\
& \text { i.e., } \log { }^{[p-1]} T_{F}\left(\exp ^{[q-1]} r\right) \geqslant \log r^{\left(\lambda_{f}(p, q)-\varepsilon\right)} \\
& \text { i.e., } \log ^{[p-2]} T_{F}\left(\exp ^{[q-1]} r\right) \geqslant r^{\left(\lambda_{f}(p, q)-\varepsilon\right)} \text {. }
\end{aligned}
$$

Now combining (9) of Case I and (13), we get for all sufficiently large values of $r$ that

$$
\begin{aligned}
& \frac{\log ^{[p-1]} T_{f \circ g}\left(\exp ^{[n-1]} r\right)}{\log ^{[p-2]} T_{F}\left(\exp ^{[q-1]} r\right)} \\
& \quad \leq \frac{\left(\rho_{f}(p, q)+\varepsilon\right) r^{\left(\rho_{g}(m, n)+\varepsilon\right)}+O(1)}{r^{\left(\lambda_{f}(p, q)-\varepsilon\right)}} .
\end{aligned}
$$

Now in view of (5) it follows from (14) that

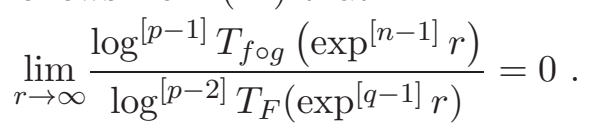

This proves the first part of the theorem.

Again combining (12) of Case II and (13), we obtain for all sufficiently large values of $r$ that

$$
\frac{\log [p+m-q-2]}{{ }^{[o g} T_{f}\left(\exp ^{[n-2]} T_{F}\left(\exp ^{[q-1]} r\right)\right.} \leq \frac{r^{\rho_{g}(m, n)+\varepsilon}+O(1)}{r^{\left(\lambda_{f}(p, q)-\varepsilon\right)}} .
$$

Now in view of (5) it follows from (15) that

$$
\lim _{r \rightarrow \infty} \frac{\log ^{[p+m-q-2]} T_{f \circ g}\left(\exp ^{[n-1]} r\right)}{\log { }^{[p-2]} T_{F}\left(\exp ^{[q-1]} r\right)}=0 .
$$

Thus the theorem follows.

Theorem 10. Let $f$ be a transcendental meromorphic function and $g$ be an entire function such that $\lambda_{g}(m, n)<\lambda_{f}(p, q) \leq \rho_{f}(p, q)<\infty$ where $p, q, m, n$ are positive integers with $p \geq q$ and $m \geq n$. Also let $F=f^{\alpha} Q[f]$ where $Q[f]$ is a differential polynomial in $f$, then for any $\alpha \geq 1$ 


$$
\text { (i) } \underset{r \rightarrow \infty}{\lim } \frac{\log ^{[p-1]} T_{f \circ g}\left(\exp ^{[n-1]} r\right)}{\log ^{[p-2]} T_{F}\left(\exp ^{[q-1]} r\right)}=0 \quad \text { if } \quad q \geq m
$$

and

$$
\text { (ii) } \underset{r \rightarrow \infty}{\lim } \frac{\log ^{[p+m-q-2]} T_{f \circ g}\left(\exp ^{[n-1]} r\right)}{\log ^{[p-2]} T_{F}\left(\exp ^{[q-1]} r\right)}=0 \quad \text { if } \quad q<m \text {. }
$$

Proof. For a sequence of values of $r$ tending to infinity that

$$
\begin{aligned}
\log ^{[m]} M_{g}\left(\exp ^{[n-1]} r\right) & \leqslant\left(\lambda_{g}(m, n)+\varepsilon\right) \log { }^{[n]} \exp ^{[n-1]} r \\
\text { i.e., } \log ^{[m]} M_{g}\left(\exp ^{[n-1]} r\right) & \leqslant \log r^{\lambda_{g}(m, n)+\varepsilon} \\
\text { i.e., } \log ^{[m-1]} M_{g}\left(\exp ^{[n-1]} r\right) & \leqslant \log r^{\lambda_{g}(m, n)+\varepsilon} .
\end{aligned}
$$

Now from (7) and (16) we get for a sequence of values of $r$ tending to infinity that

$$
\log ^{[p-1]} T_{f \circ g}\left(\exp ^{[n-1]} r\right) \leqslant\left(\rho_{f}(p, q)+\varepsilon\right) r^{\lambda_{g}(m, n)+\varepsilon}+O(1) .
$$

Combining (13) and (17) we obtain for a sequence of values of $r$ tending to infinity that

$$
\frac{\log ^{[p-1]} T_{f \circ g}\left(\exp ^{[n-1]} r\right)}{\log ^{[p-2]} T_{F}\left(\exp ^{[q-1]} r\right)} \leq \frac{\left(\rho_{f}(p, q)+\varepsilon\right) r^{\lambda_{g}(m, n)+\varepsilon}+O(1)}{r^{\left(\lambda_{f}(p, q)-\varepsilon\right)}}
$$

Now in view of (5) we have from (18) that

$$
\varliminf_{r \rightarrow \infty} \frac{\log ^{[p-1]} T_{f \circ g}\left(\exp ^{[n-1]} r\right)}{\log \left[{ }^{[p-2]} T_{F}\left(\exp ^{[q-1]} r\right)\right.}=0 .
$$

This proves the first part of the theorem.

Again for a sequence of values of $r$ tending to infinity that

$$
\begin{aligned}
\log ^{[m]} M_{g}\left(\exp ^{[n-1]} r\right) \leqslant\left(\lambda_{g}(m, n)+\varepsilon\right) \log { }^{[n]} \exp ^{[n-1]} r \\
\text { i.e., } \log ^{[m]} M_{g}\left(\exp ^{[n-1]} r\right) \leqslant \log r^{\left(\lambda_{g}(m, n)+\varepsilon\right)} \\
\text { i.e., } \exp ^{[m-q]} \log ^{[m]} M_{g}\left(\exp ^{[n-1]} r\right) \leqslant \exp ^{[m-q]} \log r^{\left(\lambda_{g}(m, n)+\varepsilon\right)} \\
\text { i.e., } \exp ^{[m-q]} \log ^{[m]} M_{g}\left(\exp ^{[n-1]} r\right) \leqslant \exp ^{[m-q-1]} r^{\left(\lambda_{g}(m, n)+\varepsilon\right)}
\end{aligned}
$$

Now from (10) and (19) we have for a sequence of values of $r$ tending to infinity that 


$$
\begin{gathered}
\log ^{[p-1]} T_{f \circ g}\left(\exp ^{[n-1]} r\right) \leqslant\left(\rho_{f}(p, q)+\varepsilon\right) \exp ^{[m-q-1]} r^{\left(\lambda_{g}(m, n)+\varepsilon\right)}+O(1) \\
\text { i.e., } \log ^{[p]} T_{f \circ g}\left(\exp ^{[n-1]} r\right) \leqslant \exp ^{[m-q-2]} r^{\left(\lambda_{g}(m, n)+\varepsilon\right)}+O(1) \\
\text { i.e., } \log ^{[p+m-q-2]} T_{f \circ g}\left(\exp ^{[n-1]} r\right) \\
\leqslant \log ^{[m-q-2]} \exp ^{[m-q-2]} r^{\left(\lambda_{g}(m, n)+\varepsilon\right)}+O(1) \\
\text { i.e., } \log ^{[p+m-q-2]} T_{f \circ g}\left(\exp ^{[n-1]} r\right) \leqslant r^{\left(\lambda_{g}(m, n)+\varepsilon\right)}+O(1)
\end{gathered}
$$

Combining (13) and (20) we obtain for a sequence of values of $r$ tending to infinity that

$$
\frac{\log ^{[p+m-q-2]} T_{f \circ g}\left(\exp ^{[n-1]} r\right)}{\log ^{[p-2]} T_{F}\left(\exp ^{[q-1]} r\right)} \leq \frac{r^{\left(\lambda_{g}(m, n)+\varepsilon\right)}+O(1)}{r^{\lambda_{f}(p, q)-\varepsilon}}
$$

Now in view of (5) it follows from (21) that

$$
\varliminf_{r \rightarrow \infty} \frac{\log ^{[p+m-q-2]} T_{f \circ g}\left(\exp ^{[n-1]} r\right)}{\log ^{[p-2]} T_{F}\left(\exp ^{[q-1]} r\right)}=0 .
$$

This establishes the second part of the theorem.

Theorem 11. Let $g$ be an entire function and $f$ be a transcendental meromorphic function such that $0<\lambda_{f}(p, q) \leq \rho_{f}(p, q)<\infty$, where $p$ and $q$ are any two positive integers with $p \geq q$. Also let $F=f^{\alpha} Q[f]$ where $Q[f]$ is a differential polynomial in $f$, then for any $\alpha \geq 1$

$$
\begin{aligned}
& \text { (i) } \varlimsup_{r \rightarrow \infty} \frac{\log ^{[p-1]} T_{f \circ g}(r)}{\log ^{[p-1]} T_{F}\left(\exp \left(r^{\mu}\right)\right)}=\infty \quad \text { if } \quad q=1 \\
& \text { (ii) } \varlimsup_{r \rightarrow \infty} \frac{\log ^{[p-1]} T_{f \circ g}(r)}{\log ^{[p-1]} T_{F}\left(\exp \left(r^{\mu}\right)\right)} \geq \frac{\beta \lambda_{f}(p, q)}{\mu \rho_{f}(p, q)} \quad \text { if } \quad q=2
\end{aligned}
$$

and

$$
\text { (iii) } \varlimsup_{r \rightarrow \infty} \frac{\log ^{[p-1]} T_{f \circ g}(r)}{\log ^{[p-1]} T_{F}\left(\exp \left(r^{\mu}\right)\right)} \geq \frac{\lambda_{f}(p, q)}{\rho_{f}(p, q)} \quad \text { if } \quad q>2,
$$

where $0<\mu<\beta<\rho_{g}$. 
Proof. Since $0<\mu<\beta<\rho_{g}$, then from Lemma 4 we obtain for a sequence of values of $r$ tending to infinity that

$$
\begin{aligned}
\log ^{[p-1]} T_{f \circ g}(r) & \geq \log ^{[p-1]} T_{f}\left(\exp \left(r^{\alpha}\right)\right) \\
\text { i.e., } \log ^{[p-1]} T_{f \circ g}(r) & \geq\left(\lambda_{f}(p, q)-\varepsilon\right) \log ^{[q]} \exp \left(r^{\alpha}\right) \\
\text { i.e., } \log ^{[p-1]} T_{f \circ g}(r) & \geq\left(\lambda_{f}(p, q)-\varepsilon\right) \log ^{[q-1]}\left(r^{\alpha}\right) .
\end{aligned}
$$

Again from the definition of $\rho_{F}(p, q)$ it follows in view of Lemma 8, for all sufficiently large values of $r$ that

$$
\begin{gathered}
\log ^{[p-1]} T_{F}\left(\exp \left(r^{\mu}\right)\right) \leq\left(\rho_{F}(p, q)+\varepsilon\right) \log ^{[q]} \exp \left(r^{\mu}\right) \\
\text { i.e., } \log ^{[p-1]} T_{F}\left(\exp \left(r^{\mu}\right)\right) \leq\left(\rho_{f}(p, q)+\varepsilon\right) \log ^{[q-1]}\left(r^{\mu}\right) .
\end{gathered}
$$

Thus from (22) and (23) we have for a sequence of values of $r$ tending to infinity that

$$
\frac{\log ^{[p-1]} T_{f \circ g}(r)}{\log ^{[p-1]} T_{F}\left(\exp \left(r^{\mu}\right)\right)} \geq \frac{\left(\lambda_{f}(p, q)-\varepsilon\right) \log ^{[q-1]}\left(r^{\alpha}\right)}{\left(\rho_{f}(p, q)+\varepsilon\right) \log ^{[q-1]}\left(r^{\mu}\right)} .
$$

Since $\mu<\beta$, the theorem follows from (24).

Theorem 12. Let $f$ be a transcendental meromorphic function and $g$ be an entire function such that $0<\lambda_{f}(p, q) \leq \rho_{f}(p, q)<\infty$ and $\rho_{g}(m, n)$ $<\infty$ where $p, q, m, n$ are positive integers with $p \geq q$ and $m \geq n$. Also let $F=f^{\alpha} Q[f]$ where $Q[f]$ is a differential polynomial in $f$, then for any $\alpha \geq 1$

$$
\text { (i) } \varlimsup_{r \rightarrow \infty} \frac{\log ^{[p]} T_{f \circ g}\left(\exp ^{[n-1]} r\right)}{\log ^{[p-1]} T_{F}\left(\exp ^{[q-1]} r\right)} \leqslant \frac{\rho_{g}(m, n)}{\lambda_{f}(p, q)} \quad \text { if } \quad q \geq m
$$

and

$$
\text { (ii) } \varlimsup_{r \rightarrow \infty} \frac{\log ^{[p+m-q-1]} T_{f \circ g}\left(\exp ^{[n-1]} r\right)}{\log ^{[p-1]} T_{F}\left(\exp ^{[q-1]} r\right)} \leqslant \frac{\rho_{g}(m, n)}{\lambda_{f}(p, q)} \quad \text { if } \quad q<m \text {. }
$$

Proof. In view of Lemma 8, we have for all sufficiently large values of $r$ that

$$
\begin{aligned}
\log ^{[p-1]} T_{F}\left(\exp ^{[q-1]} r\right) & \geq\left(\lambda_{F}(p, q)-\varepsilon\right) \log [q] \exp ^{[q-1]} r \\
\text { i.e., } \quad \log ^{[p-1]} T_{F}\left(\exp ^{[q-1]} r\right) & \geq\left(\lambda_{f}(p, q)-\varepsilon\right) \log r .
\end{aligned}
$$

Case I. If $q \geqslant m$, then from (9) and (25) we get for all sufficiently large values of $r$ that

$$
\frac{\log ^{[p]} T_{f \circ g}\left(\exp ^{[n-1]} r\right)}{\log ^{[p-1]} T_{F}\left(\exp ^{[q-1]} r\right)} \leqslant \frac{\left(\rho_{g}(m, n)+\varepsilon\right) \log r+O(1)}{\left(\lambda_{f}(p, q)-\varepsilon\right) \log r} .
$$


Since $\varepsilon(>0)$ is arbitrary, it follows from above that

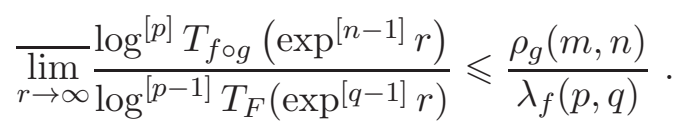

This proves the first part of the theorem.

Case II. If $q<m$ then from (12) and (25) we obtain for all sufficiently large values of $r$ that

$$
\frac{\log ^{[p+m-q-1]} T_{f \circ g}\left(\exp ^{[n-1]} r\right)}{\log ^{[p-1]} T_{F}\left(\exp ^{[q-1]} r\right)} \leqslant \frac{\left(\rho_{g}(m, n)+\varepsilon\right) \log r+O(1)}{\left(\lambda_{f}(p, q)-\varepsilon\right) \log r} .
$$

As $\varepsilon(>0)$ is arbitrary, it follows from above that

$$
\varlimsup_{r \rightarrow \infty} \frac{\log ^{[p+m-q-1]} T_{f \circ g}\left(\exp ^{[n-1]} r\right)}{\log ^{[p-1]} T_{F}\left(\exp ^{[q-1]} r\right)} \leqslant \frac{\rho_{g}(m, n)}{\lambda_{f}(p, q)} .
$$

Thus the second part of the theorem is established.

Theorem 13. Let $f$ be meromorphic and $g$ be a transcendental entire such that $\rho_{f}(p, q)$ and $\lambda_{g}^{[l]}$ are both finite where $p, q, l$ are positive integers with $p>q$ and $l \geq 2$. Also let $G=g^{\alpha} Q[g]$ where $Q[g]$ is a differential polynomial in $g$, then for any $\alpha \geq 1$

$$
\varliminf_{r \rightarrow \infty} \frac{\log ^{[p-1]} T_{f \circ g}(r)}{\log ^{[l-2]} T_{G}(r)} \leqslant \rho_{f}(p, q) \cdot 2^{\lambda_{g}^{[l]}} \quad \text { if } q \geq l-1>1,
$$

$$
\varliminf_{r \rightarrow \infty} \frac{\log ^{[p+l-q-2]} T_{f \circ g}(r)}{\log ^{[l-2]} T_{G}(r)} \leqslant 2^{\lambda_{g}^{[l]}} \text { if } q<l-1,
$$

and

$$
\text { (iii) } \quad \underline{\lim } \frac{\log ^{[p-1]} T_{f \circ g}(r)}{T_{G}(r)} \leqslant 3 \beta \cdot \rho_{f}(p, q) \cdot 2^{\lambda_{g}} \quad \text { if } \quad q \geq l-1=1 \text {, }
$$

where $\beta>1$.

Proof. As $\varepsilon(>0)$ is arbitrary and $T_{g}(r) \leqslant \log ^{+} M_{g}(r)\{\mathrm{cf}$. [5]\}, we have from Lemma 3 for all sufficiently large values of $r$ that

$$
\log ^{[p-1]} T_{f \circ g}(r) \leqslant\left(\rho_{f}(p, q)+\varepsilon\right) \log ^{[q]} M_{g}(r)+O(1) .
$$


Case I. Let $q \geq l-1$. Then from (26) we obtain for all sufficiently large values of $r$ that

$$
\log ^{[p-1]} T_{f \circ g}(r) \leqslant\left(\rho_{f}(p, q)+\varepsilon\right) \log ^{[l-1]} M_{g}(r)+O(1) .
$$

Since $\varepsilon(>0)$ we get from above that

$$
\varliminf_{r \rightarrow \infty} \frac{\log ^{[p-1]} T_{f \circ g}(r)}{\log ^{[l-2]} T_{G}(r)} \leqslant \rho_{f}(p, q) \cdot \underline{\lim _{r \rightarrow \infty}} \frac{\log ^{[l-1]} M_{g}(r)}{\log ^{[l-2]} T_{G}(r)} .
$$

Case II. Let $q<l-1$. Then from (26) we get for all sufficiently large values of $r$ that

$$
\begin{gathered}
\log ^{[l-q-1]} \log ^{[p-1]} T_{f \circ g}(r) \leqslant \log ^{[l-q-1]}\left\{\left(\rho_{f}(p, q)+\varepsilon\right) \log ^{[q]} M_{g}(r)+O(1)\right\} \\
\text { i.e., } \quad \log ^{[p+l-q-2]} T_{f \circ g}(r) \leqslant \log ^{[l-1]} M_{g}(r)+O(1) \\
\text { i.e., } \quad \frac{\log ^{[p+l-q-2]} T_{f \circ g}(r)}{\log ^{[l-2]} T_{G}(r)} \leqslant \frac{\log ^{[l-1]} M_{g}(r)+O(1)}{\log ^{[l-2]} T_{G}(r)} .
\end{gathered}
$$

Therefore we get from above that

$$
\varliminf_{r \rightarrow \infty} \frac{\log ^{[p+l-q-2]} T_{f \circ g}(r)}{\log ^{[l-2]} T_{G}(r)} \leqslant \underline{\lim } \frac{\log ^{[l-1]} M_{g}(r)}{\log ^{[l-2]} T_{G}(r)} .
$$

Now let $l>2$. Since $\varliminf_{r \rightarrow \infty} \frac{\log ^{[l-2]} T_{g}(r)}{r^{\lambda_{g}^{l]}(r)}}=1$, for given $\varepsilon(0<\varepsilon<1)$ we get for a sequence of values of $r$ tending to infinity that

$$
\log ^{[l-2]} T_{g}(r)<(1+\varepsilon) r^{\lambda_{g}^{[l]}(r)}
$$

and for all sufficiently large values of $r$,

$$
\log ^{[l-2]} T_{g}(r)>(1-\varepsilon) r^{\lambda_{g}^{[l]}(r)} .
$$

Since $\log M_{g}(r) \leq 3 T_{g}(2 r)\{\mathrm{cf}$. [5] $\}$ and $T_{g}(r)=O\left\{T_{G}(r)\right\}$ as $r \rightarrow \infty\{\mathrm{cf}$. $[3]\}$, we get for a sequence of values of $r$ tending to infinity and for any $\delta(>0)$ that

$$
\begin{aligned}
& \frac{\log ^{[l-1]} M_{g}(r)}{\log ^{[l-2]} T_{G}(r)}<\frac{\log ^{[l-1]} M_{g}(r)}{\log ^{[l-2]} T_{g}(r)+O(1)}<\frac{\log ^{[l-2]} T_{g}(2 r)+O(1)}{\log ^{[l-2]} T_{g}(r)+O(1)} \\
& <\frac{(1+\varepsilon)}{(1-\varepsilon)} \cdot \frac{(2 r)^{\lambda_{g}^{[l]}+\delta}}{(2 r)^{\lambda_{g}^{[l]}+\delta-\lambda_{g}^{[l]}(2 r)}} \cdot \frac{1}{r^{\lambda_{g}^{[l]}(r)}}+O(1) \\
& <\frac{(1+\varepsilon)}{(1-\varepsilon)} \cdot 2^{\lambda_{g}^{[l]}+\delta}+O(1)
\end{aligned}
$$


because $r^{\lambda_{g}^{[l]}+\delta-\lambda_{g}^{[l]}(r)}$ is ultimately an increasing function of $r$ by Lemma 5 .

Since $\varepsilon(>0)$ and $\delta(>0)$ are both arbitrary, we get from above that

$$
\varliminf_{r \rightarrow \infty} \frac{\log ^{[l-1]} M_{g}(r)}{\log ^{[l-2]} T_{G}(r)} \leq 2^{\lambda_{g}^{[l]}} .
$$

Again let $l=2$. Since $\varliminf_{r \rightarrow \infty} \frac{T_{g}(r)}{r^{\lambda}(r)}=1$, in view of condition (v) of Definition 1 it follows for a sequence of values of $r$ tending to infinity and for a given $\varepsilon(0<\varepsilon<1)$ that

$$
T_{g}(r)<(1+\varepsilon) r^{\lambda_{g}(r)}
$$

and for all large positive numbers of $r$,

$$
T_{g}(r)>(1-\varepsilon) r^{\lambda_{g}(r)} .
$$

As $\log M_{g}(r) \leq 3 T_{g}(2 r)\{$ cf. [5] $\}$ and $T_{g}(r)=O\left\{T_{G}(r)\right\}$ as $r \rightarrow \infty\{$ cf. [3] $\}$, we get for any $\delta(>0), \beta>1$ and for a sequence of values of $r$ tending to infinity that

$$
\begin{aligned}
\frac{\log M_{g}(r)}{T_{G}(r)} & <\beta \cdot \frac{\log M_{g}(r)}{T_{g}(r)} \\
\text { i.e., } \frac{\log M_{g}(r)}{T_{G}(r)} & <\beta \cdot \frac{3(1+\varepsilon)}{(1-\varepsilon)} \cdot \frac{(2 r)^{\lambda_{g}+\delta}}{(2 r)^{\lambda_{g}+\delta-\lambda_{g}(2 r)}} \cdot \frac{1}{r^{\lambda_{g}(r)}}+O(1) \\
\text { i.e., } \frac{\log M_{g}(r)}{T_{G}(r)} & <\frac{3 \beta(1+\varepsilon)}{(1-\varepsilon)} \cdot 2^{\lambda_{g}+\delta}+O(1),
\end{aligned}
$$

because $r^{\lambda_{g}+\delta-\lambda_{g}(r)}$ is ultimately an increasing function of $r$ by Lemma 5 . Since $\varepsilon(>0)$ and $\delta(>0)$ are both arbitrary, we get from (30) that

$$
\varliminf_{r \rightarrow \infty} \frac{\log M_{g}(r)}{T_{G}(r)} \leq 3 \beta \cdot 2^{\lambda_{g}} .
$$

Therefore from (27) of Case I and (29) it follows that

$$
\varliminf_{r \rightarrow \infty} \frac{\log ^{[p-1]} T_{f \circ g}(r)}{\log ^{[l-2]} T_{G}(r)} \leqslant \rho_{f}(p, q) \cdot 2^{\lambda_{g}^{[l]}} .
$$

This proves the first part of the theorem. Also from (28) of Case II and (29) we obtain that

$$
\varliminf_{r \rightarrow \infty} \frac{\log ^{[p+l-q-2]} T_{f \circ g}(r)}{\log ^{[l-2]} T_{G}(r)} \leqslant 2^{\lambda_{g}^{[l]}} .
$$

Thus the second part of the theorem is established. Again putting $l=2$ in (27) of Case I and in view of (31) we obtain that 


$$
\varliminf_{r \rightarrow \infty} \frac{\log [p-1]}{T_{f \circ g}(r)} \leqslant 3 \beta \cdot \rho_{f}(p, q) \cdot 2^{\lambda g} .
$$

Thus the third part of the theorem follows.

Corollary 14. Under the same conditions of Theorem 13, if $l=2$ then

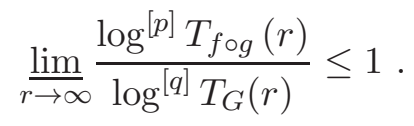

Proof. If $q \geq 1$, then from (26) we obtain for all sufficiently large values of $r$ that

$$
\log ^{[p]} T_{f \circ g}(r) \leqslant \log ^{[q+1]} M_{g}(r)+O(1) .
$$

Now from (30) we have for a sequence of values of $r$ tending to infinity that

$$
\begin{aligned}
\log M_{g}(r) & \leq\left\{\frac{3 \beta(1+\varepsilon)}{(1-\varepsilon)} \cdot 2^{\lambda_{g}+\delta}\right\} \cdot T_{G}(r) \\
\text { i.e., } \log ^{[q+1]} M_{g}(r) & \leq \log { }^{[q]} T_{G}(r)+O(1) .
\end{aligned}
$$

Now combining (32) and (33) it follows for a sequence of values of $r$ tending to infinity that

$$
\begin{aligned}
& \log ^{[p]} T_{f \circ g}(r) \leqslant \log { }^{[q]} T_{G}(r)+O(1) \\
& \text { i.e., } \frac{\log ^{[p]} T_{f \circ g}(r)}{\log ^{[q]} T_{G}(r)} \leq 1+\frac{O(1)}{\log [q]} T_{G}(r)
\end{aligned}
$$

So from above we obtain that

$$
\varliminf_{r \rightarrow \infty} \frac{\log ^{[p]} T_{f \circ g}(r)}{\log ^{[q]} T_{G}(r)} \leq 1
$$

Thus the corollary follows.

Theorem 15. Let $f$ be meromorphic and $g$ be a transcendental entire such that $\rho_{f}(p, q)$ and $\rho_{g}^{[l]}$ are finite where $p, q, l$ are positive integers with $p>q$ and $l \geq 2$. Also let $G=g^{\alpha} Q[g]$, where $Q[g]$ is a differential polynomial in $g$, then for any $\alpha \geq 1$

$$
\text { (i) } \quad \varliminf_{r \rightarrow \infty} \frac{\log ^{[p-1]} T_{f \circ g}(r)}{\log ^{[l-2]} T_{G}(r)} \leqslant \rho_{f}(p, q) \cdot 2^{\rho_{g}^{[l]}} \quad \text { if } q \geq l-1>1
$$


(ii) $\quad \varliminf_{r \rightarrow \infty} \frac{\log ^{[p+l-q-2]} T_{f \circ g}(r)}{\log ^{[l-2]} T_{G}(r)} \leqslant 2^{\rho_{g}^{[l]}}$ if $q<l-1$

and

$$
\text { (iii) } \quad \varliminf_{r \rightarrow \infty} \frac{\log [p-1]}{T_{f \circ g}(r)} \leqslant 3 \beta \cdot \rho_{f}(p, q) \cdot 2^{\rho_{g}} \quad \text { if } q \geq l-1=1 \text {, }
$$

where $\beta>1$.

Proof. Case I. Let $l>2$. As $\varlimsup_{r \rightarrow \infty} \frac{\log ^{[l-2]} T_{g}(r)}{r^{\rho_{g}^{l l]}}(r)}=1$, for given $\varepsilon(0<\varepsilon<1)$ we obtain for all sufficiently large values of $r$ that

$$
\log ^{[l-2]} T_{g}(r)<(1+\varepsilon) r^{\rho_{g}^{[l]}(r)}
$$

and for a sequence of values of $r$ tending to infinity,

$$
\log ^{[l-2]} T_{g}(r)>(1-\varepsilon) r^{\rho_{g}^{[l]}(r)} .
$$

Since $\log M_{g}(r) \leq 3 T_{g}(2 r)\{$ cf. [5] $\}$ and $T_{g}(r)=O\left\{T_{G}(r)\right\}$ as $r \rightarrow \infty\{$ cf. [3] $\}$, for a sequence of values of $r$ tending to infinity we get for any $\delta(>0)$ that

$$
\begin{aligned}
\frac{\log ^{[l-1]} M_{g}(r)}{\log ^{[l-2]} T_{G}(r)} & <\frac{\log ^{[l-1]} M_{g}(r)}{\log ^{[l-2]} T_{g}(r)+O(1)}<\frac{\log ^{[l-2]} T_{g}(2 r)+O(1)}{\log { }^{[l-2]} T_{g}(r)+O(1)} \\
& <\frac{(1+\varepsilon)}{(1-\varepsilon)} \cdot \frac{(2 r)^{\rho_{g}^{[l]}+\delta}}{(2 r)^{\rho_{g}^{[l]}+\delta-\rho_{g}^{[l]}(2 r)}} \cdot \frac{1}{r^{\rho_{g}^{[l]}(r)}}+O(1) \\
& <\frac{(1+\varepsilon)}{(1-\varepsilon)} \cdot 2^{\rho_{g}^{[l]}+\delta}
\end{aligned}
$$

because $r^{\rho_{g}^{[l]}+\delta-\rho_{g}^{[l]}(r)}$ is ultimately an increasing function of $r$ by Lemma 6 .

Since $\varepsilon(>0)$ and $\delta(>0)$ are both arbitrary, we get from above that

$$
\varliminf_{r \rightarrow \infty} \frac{\log ^{[l-1]} M_{g}(r)}{\log ^{[l-2]} T_{G}(r)} \leq 2^{\rho_{g}^{[l]}} .
$$

Case II. Let $l=2$. Since $\varlimsup_{r \rightarrow \infty} \frac{T_{g}(r)}{r^{\rho g}(r)}=1$, in view of condition (v) of Definition 2 it follows for all sufficiently large values of $r$ and for a given $\varepsilon(0<\varepsilon<1)$ that

$$
T_{g}(r)<(1+\varepsilon) r^{\rho_{g}(r)}
$$

and for a sequence of values of $r$ tending to infinity

$$
T_{g}(r)>(1-\varepsilon) r^{\rho_{g}(r)} .
$$


As $\log M_{g}(r) \leq 3 T_{g}(2 r)\{$ cf. [5] $\}$ and $T_{g}(r)=O\left\{T_{G}(r)\right\}$ as $r \rightarrow \infty$ cf. [3]\}, we get for any $\delta(>0), \beta>1$ and for a sequence of values of $r$ tending to infinity that

$$
\begin{aligned}
\frac{\log M_{g}(r)}{T_{G}(r)} & <\beta \cdot \frac{\log M_{g}(r)}{T_{g}(r)} \\
\text { i.e., } \frac{\log M_{g}(r)}{T_{G}(r)} & <\beta \cdot \frac{3(1+\varepsilon)}{(1-\varepsilon)} \cdot \frac{(2 r)^{\rho_{g}+\delta}}{(2 r)^{\rho_{g}+\delta-\rho_{g}(2 r)}} \cdot \frac{1}{r^{\rho_{g}(r)}}+O(1) \\
\text { i.e., } \frac{\log M_{g}(r)}{T_{G}(r)} & <\frac{3 \beta(1+\varepsilon)}{(1-\varepsilon)} \cdot 2^{\rho_{g}+\delta}+O(1)
\end{aligned}
$$

because $r^{\rho_{g}+\delta-\rho_{g}(r)}$ is ultimately an increasing function of $r$ by Lemma 6 .

Since $\varepsilon(>0)$ and $\delta(>0)$ are both arbitrary, we get from the above that

$$
\varliminf_{r \rightarrow \infty} \frac{\log M_{g}(r)}{T_{G}(r)} \leq 3 \cdot 2^{\rho_{g}} .
$$

Therefore from (27) and (34) it follows that

$$
\varliminf_{r \rightarrow \infty} \frac{\log ^{[p-1]} T_{f \circ g}(r)}{\log ^{[l-2]} T_{G}(r)} \leqslant \rho_{f}(p, q) \cdot 2^{\rho_{g}^{[l]}} .
$$

This proves the first part of the theorem. Similarly from (28) and (34) we get

$$
\varliminf_{r \rightarrow \infty} \frac{\log ^{[p+l-q-2]} T_{f \circ g}(r)}{\log ^{[l-2]} T_{G}(r)} \leqslant 2^{\rho_{g}^{[l]}} .
$$

Thus the second part of the theorem follows.

Again putting $l=2$ in (27) and in view of (35) we obtain that

$$
\varliminf_{r \rightarrow \infty} \frac{\log ^{[p-1]} T_{f \circ g}(r)}{T_{G}(r)} \leqslant 3 \beta \cdot \rho_{f}(p, q) \cdot 2^{\rho g} .
$$

Thus the third part of the theorem is established.

Theorem 16. Let $f$ be a transcendental meromorphic function and $g$ be an entire function such that $\rho_{f}(p, q)<\infty$ and $\lambda_{f \circ g}(p, q)=\infty$ where $p$ and $q$ are positive integers with $p \geq q$. Also let $F=f^{\alpha} Q[f]$ where $Q[f]$ is a differential polynomial in $f$, then for any $\alpha \geq 1$

$$
\lim _{r \rightarrow \infty} \frac{\log ^{[p]} T_{f \circ g}(r)}{\log ^{[p]} T_{F}\left(r^{A}\right)}=\infty,
$$

where $A(>0)$ is any positive number. 
Proof. If possible, let there exists a constant $\beta$ such that for a sequence of positive numbers of $r$ tending to infinity we have

$$
\log ^{[p]} T_{f \circ g}(r) \leq \beta \cdot \log ^{[p]} T_{F}\left(r^{A}\right) .
$$

Again from the definition of $\rho_{F}(p, q)$ and in view of Lemma 8, it follows for all sufficiently large positive numbers of $r$ that

$$
\begin{gathered}
\log ^{[p]} T_{F}\left(r^{A}\right) \leq\left(\rho_{F}(p, q)+\varepsilon\right) \log ^{[q]} r+O(1) . \\
\text { i.e., } \log ^{[p]} T_{F}\left(r^{A}\right) \leq\left(\rho_{f}(p, q)+\varepsilon\right) \log ^{[q]} r+O(1) .
\end{gathered}
$$

Now combining (36) and (37) we obtain for a sequence of positive numbers of $r$ tending to infinity that

$$
\begin{aligned}
\log ^{[p]} T_{f \circ g}(r) & \leq \beta \cdot\left(\rho_{f}(p, q)+\varepsilon\right) \log ^{[q]} r+O(1) \\
\text { i.e., } \lambda_{f \circ g}(p, q) & \leq \beta \cdot\left(\rho_{f}(p, q)+\varepsilon\right),
\end{aligned}
$$

which contradicts the condition $\lambda_{f \circ g}(p, q)=\infty$. So for all sufficiently large positive numbers of $r$ we get that

$$
\log ^{[p]} T_{f \circ g}(r) \geq \beta \cdot \log ^{[p]} T_{F}\left(r^{A}\right),
$$

from which the theorem follows.

In the line of Theorem 16, one can easily prove the following theorem and therefore its proof is omitted.

Theorem 17. Let $f$ be meromorphic function and $g$ be a transcendental entire function such that $\rho_{g}(p, q)<\infty$ and $\lambda_{f \circ g}(p, q)=\infty$ where $p$ and $q$ are positive integers with $p \geq q$. Also let $G=g^{\alpha} Q[g]$ where $Q[g]$ is a differential polynomial in $g$, then for any $\alpha \geq 1$

$$
\lim _{r \rightarrow \infty} \frac{\log ^{[p]} T_{f \circ g}(r)}{\log ^{[p]} T_{G}\left(r^{A}\right)}=\infty,
$$

where $A(>0)$ is any positive number.

Remark 1. Theorem 16 is also valid with "limit superior" instead of "limit" if $\lambda_{f \circ g}(p, q)=\infty$ is replaced by $\rho_{f \circ g}(p, q)=\infty$ and the other conditions remain the same.

Remark 2. Theorem 17 is also valid with "limit superior" instead of "limit" if $\lambda_{f \circ g}(p, q)=\infty$ is replaced by $\rho_{f \circ g}(p, q)=\infty$ and the other conditions remain the same. 
Corollary 18. Under the assumptions of Theorem 16 and Remark 1,

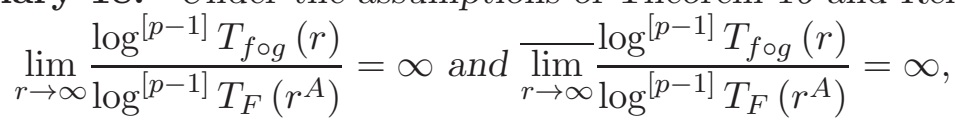

respectively.

Proof. By Theorem 16 we obtain for all sufficiently large values of $r$ and for $K>1$,

$$
\begin{aligned}
\log ^{[p]} T_{f \circ g}(r) & \geq K \cdot \log ^{[p]} T_{F}\left(r^{A}\right) \\
\text { i.e., } \log ^{[p-1]} T_{f \circ g}(r) & \geq\left\{\log ^{[p-1]} T_{F}\left(r^{A}\right)\right\}^{K},
\end{aligned}
$$

from which the first part of the corollary follows.

Similarly, using Remark 1, we obtain the second part of the corollary.

Corollary 19. Under the assumptions of Theorem 17 and Remark 2,

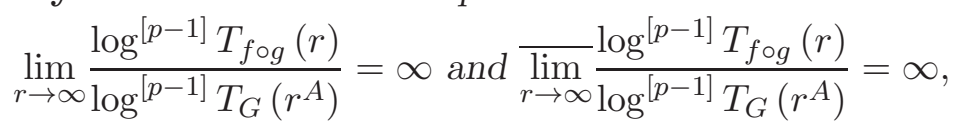

respectively.

In the line of Corollary 18, one can easily verify Corollary 19 with the help of Theorem 17 and Remark 2 respectively and therefore its proof is omitted.

\section{Acknowledgment}

The author is thankful to Prof. Virginia Kiryakova for her valuable suggestions which considerably improved the presentation of the paper.

\section{References}

[1] W. Bergweiler, On the Nevanlinna Characteristic of a composite function, Complex Variables Theory Appl., 10, No 2-3 (1988), 225-236.

[2] W. Bergweiler, On the growth rate of composite meromorphic functions, Complex Variables Theory Appl., 14, No 1-4 (1990), 187-196.

[3] S.S. Bhooshnurmath, K.S.L.N. Prasad, The value distribution of some differential polynomials, Bull. Cal. Math. Soc., 101, No 1 (2009), 55-62. 
[4] S.K. Datta, T. Biswas, Growth rates of composite entire and meromorphic functions, Bull. Allahabad Math. Soc., 27 (2012), 55-94.

[5] W.K. Hayman, Meromorphic Functions, The Clarendon Press, Oxford (1964).

[6] O.P. Juneja, G.P. Kapoor, S.K. Bajpai, On the $(p, q)$-order and lower $(p, q)$ order of an entire function, J. Reine Angew. Math., 282 (1976), 53-67.

[7] Q. Lin, C. Dai, On a conjecture of Shah concerning small functions, Kexue Tong bao (English Ed.), 31, No 4 (1986), 220-224.

[8] I. Lahiri, Generalised proximate order of meromorphic functions, Mat. Vesnik, 41 (1989), 9-16.

[9] I. Laine, Nevanlinna Theory and Complex Differential Equations, De Gruyter, Berlin (1993).

[10] D. Sato, On the rate of growth of entire functions of fast growth, Bull. Amer. Math. Soc., 69 (1963), 411-414.

[11] C.C. Yang, H.X. Yi, Uniqueness Theory of Meromorphic Functions, Mathematics and its Applications, 557, Kluwer Academic Publishers Group, Dordrecht (2003).

[12] L. Yang, Value Distribution Theory, Springer-Verlag, Berlin (1993).

[13] G. Valiron, Lectures on the General Theory of Integral Functions, Chelsea Publishing Company (1949). 